\title{
Retrospective evaluation of the correlation of nodules detected to have follicular neoplasm suspicion on thyroid ultrasonography with cytopathological and histopathological results
}

\author{
Gozde ERTUNC'1, Recep Gokhan ICOZ², Suha OZBEK ${ }^{3}$ \\ ${ }^{1}$ Department of Surgical Oncology, Ankara City Hospital, Ankara, Turkey \\ ${ }^{2}$ Division Endocrine Surgery, Department of General Surgery, Ege University School of Medicine Hospital, İzmir, Turkey \\ ${ }^{3}$ Department of Radiology, Ege University School of Medicine Hospital, İzmir, Turkey \\ Correspondence \\ Gozde ERTUNC \\ Ankara City Hospital, Surgical Oncology Department, Ankara, Turkey \\ e-mail:gzdrtnc@gmail.com
}

\begin{abstract}
Thyroid nodules are pathologies that are frequently encountered in general population and sometimes difficult to manage for patients and physicians. The most appropriate method for the management of these nodules is follow-up with ultrasonography (US) and fine-needle aspiration. This study investigated the correlation of suspicious nodules detected on US with cytopathological and histopathological results. Nodule size, nature, echo structure and blood supply, presence of peripheral halo, and presence of microcalcification in the nodule were compared in terms of malignancy and follicular patterns in a total of 55 patients. Statistically significant results were not found; however, we found similar rates to the literature in terms of the pathological diagnoses of malignancy and follicular pattern in patients suspected to have follicular neoplasia, which may be due to our hospital being an experienced center. We also think that further studies conducted with a higher number of patients and data on this subject can greatly contribute to the treatment and follow-up of thyroid cancer.
\end{abstract}

Key words: thyroid nodules, thyroid ultrasonography, FNAB of thyroid nodules

\section{INTRODUCTION}

Thyroid nodules are detected in a general population at a rate of $4 \%-7 \%$ by palpation and $13 \%-67 \%$ by ultrasonography (US). However, only less than $7 \%$ of these nodules are malignant (1). The clinical importance of these nodules remains unknown, and the nodule management often creates a dilemma for both physicians and patients (2).

Thyroid US and color Doppler US (CDUS) are easily available, inexpensive, noninvasive, and effective diagnostic methods that show normal thyroid anatomy and pathological structures very well without the need for radiation exposure during the process (3). Potential thyroid malignancy predictors detected by US include irregular nodule borders, hypoechogenicity, absence of a halo, rate of predominant solid components, anteroposterior nodule diameter/ transverse diameter $>1$, increased blood supply to the nodule, and calcification (4).

A US-guided fine-needle aspiration biopsy (FNAB) is a well-established method for diagnosing thyroid cancer, and most FNAB findings are sufficient for a cytological diagnosis (4). The American Thyroid Association 2015 guidelines classified biopsy results as Bethesda 1-6 indicating a malignancy risk of $1 \%-4 \%, 0 \%-3 \%, 5 \%-15 \%, 15 \%-30 \%, 60 \%-75 \%$, and $97 \%-99 \%$, respectively $(5)$.

The major histopathological features of follicular-patterned lesions are follicular-patterned growth and the presence of follicles consisting of various amounts of colloids at the center lumen, which can be visualized under a light microscope. The follicular-patterned lesions are divided into two main categories as follows: benign nodular hyperplasia and neoplastic follicular-patterned lesions, which include follicular adenoma, follicular carcinoma, and follicular variant papillary carcinoma (6).

This study aimed to determine the correlation of pathological features with cytopathological and histopathological findings in patients who underwent FNAB or surgery due to the detection of nodules with 
follicular neoplasm suspicion on thyroid US, to investigate the contribution of thyroid ultrasonography to diagnosis, and to explore the possibility of early diagnosis and treatment of thyroid cancer based on the determination of specificity and sensitivity values.

\section{MATERIAL AND METHODS}

The patients admitted to the endocrinology and endocrine surgery department of Ege University Medical Faculty Hospital between December 2010 and December 2017, who were found to have nodules with follicular neoplasm suspicion on thyroid US, were screened retrospectively, and those who underwent FNAB or surgery (total thyroidectomy, lobectomy) were included in the study. We carefully confirmed that the thyroid US examinations and pathological evaluations of the patients were reported by the radiology department and finalized by the pathology department of the hospital. Age or sex classification was not used in the selection of patients. Epidemiological and laboratory data were accessed through the database of the hospital. A total of 55 patients were included in the study. The US and CDUS examinations of the patients included in the study were performed with Siemens Acuson S1000 and S2000 devices (Siemens Acuson Solutions, CA, USA) using 9L4 (4-9 mHz) and 14L5 (5-14 $\mathrm{mHz})$ linear probes. Patients who did not undergo an ultrasonographic examination at Ege University Radiology Department were not included in the study. All ultrasonographic examinations were performed by the same physician, Prof Dr Sureyya Ozbek, at Ege University Radiology Department.

Thyroid nodules were evaluated according to the following six criteria based on the US/CDUS findings: nodule size, nodule nature, nodule echo structure, nodule blood supply, presence of a peripheral halo, and presence of microcalcification within the nodule. For nodule size, the largest millimetric value of the nodule measured from the three planes was included in the study. In terms of their nature, the nodules were divided into two groups: solid and solid-cystic (mixed). Nodules containing millimetric cystic degeneration areas and those considered to be more than $75 \%$ solid were included in the solid group, while nodules with $50 \%$ or less solidity were included in the solid-cystic group. In the evaluation of nodule echogenicity, the nodules were classified as hypoechoic, hyperechoic, and isoechoic according to the nodular thyroid parenchyma. These two parameters were assessed according to the US findings of the patients in the sample. The blood supply of the nodule was classified as increased central and peripheral blood flow. The presence of a peripheral halo referred to the observation of a complete halo around the nodule. If no halo was present around the nodule or the halo was interrupted, this was evaluated as the absence of a peripheral halo. The presence of microcalcification was defined as punctate calcification $(2 \mathrm{~mm}$ or less in diameter) with posterior acoustic shadowing and coarse calcification $(>2 \mathrm{~mm}$ ). The nodules were also classified into two groups as those with and without microcalcification.

The invasive procedures applied to the patients were divided into three groups: FNAB, total thyroidectomy, and lobectomy. Examination of the pathology results was undertaken by evaluating the cytopathology results for FNAB and the histopathology results for total thyroidectomy and lobectomy. All pathology results were examined in two groups as benign and malignant and in a further two groups based on the presence or absence of a follicular pattern. The significance rates of the cytopathological and histopathological results were evaluated separately. All US data were compared between the malignant and benign groups and between the groups with and without a follicular pattern.

The six parameters defined earlier were examined by the US and CDUS examinations, and the relationship of these parameters with the pathology results was evaluated. For statistical analyses, the IBM SPSS package program, v. 18, was used. Frequency (percentage) values were used for categorical variables, and mean \pm standard deviation [median (minimum-maximum)] values for numerical variables. The chi-square test was used to compare categorical variables in the pathological groups, and the MannWhitney $U$ test was conducted to compare numerical variables in the two groups. A $P$ value $<0.05$ indicated a statistically significant difference.

\section{RESULTS}

Fifty-five patients with nodules that were detected to have follicular neoplasm suspicion on US were included in the study. The age range was 21-77 (mean $47.8 \pm 13.8$ ) years, and the male/female gender ratio 
was $45 / 10$ (81.8\%/18.2\%). The number of patients diagnosed through FNAB and surgery was 31 (56.4\%), and 24 (43.6\%) (total thyroidectomy, $n=23$; thyroid lobectomy, $n=1)$. In total, $23(41.8 \%)$ patients were found to have malignant nodules and 32 (58.1\%) had benign nodules. No statistically significant difference was found between malignant and benign nodules.

The histopathological results of 24 patients who underwent surgery were as follows: follicular carcinoma in 1, follicular adenoma in 1, follicular nodular diseases in 2, and papillary carcinoma in 20 (follicular variant in 15 and less differentiated variant, microfollicular variant, solid variant, oncocytic variant, and classical type in 1 patient each).

Of the 24 patients who underwent surgery, 20 (80.3\%) were observed to have a follicular pattern histopathologically, which was found to be statistically significant $(P<0.05)$. Of the 31 patients who applied FNAB, $3(9.6 \%)$ were observed to have a follicular pattern histopathologically, which was found to be statis- tically significant $(P<0.05)$ (Table 1$)$.

The cytopathological results of 31 patients who underwent FNAB were as follows: non-diagnostic cytology (Bethesda 1) ( $n=1)$, follicular nodular disease (Bethesda 2) ( $n=1)$, suspicious for follicular neoplasm (SFN) (Bethesda 4) ( $n=2)$, and benign cytology (Bethesda 2) ( $n=27)$. In total, a follicular pattern was detected in 3 of 31 (9.6\%) patients who underwent FNAB. The nodule size was examined in two groups as malignant and benign; the mean size was calculated as $23.3 \mathrm{~mm}$ for the benign nodules and $26.1 \mathrm{~mm}$ for the malignant nodules, indicating no statistically significant difference $(P=0.72)$. Also, no statistically significant relationship was observed between nodule size and pathological presence of a follicular pattern (29.5 mm for follicular pattern+, $26.9 \mathrm{~mm}$ follicular pattern-) $(P=0.55)$. In addition, there is no statistically significant difference was found between US/CDUS findings and benign/malignant and follicular patterns (Table 2).

Table 1 Presence of a follicular pattern in FNAB and surgery groups

\begin{tabular}{lcccc}
\hline Groups & Surgery & FNAB & Total \\
& $N(\%)$ & $N(\%)$ & $N$ & P value \\
\hline Follicular patern $(+)$ & $20(80.3)$ & $3(9.6)$ & 23 & 32 \\
Follicular patern (-) & $4(19.7)$ & $28(90.4)$ & 32 \\
\hline
\end{tabular}

$P<0.05$

Table 2 Comparison of ultrasonographic nodule findings according to the presence of malignancy and a follicular pattern

\begin{tabular}{|c|c|c|c|c|c|c|}
\hline US/CDUS findings & Benign & Malignant & $P$ & $\begin{array}{l}\text { Follicular } \\
\text { pattern (+) }\end{array}$ & $\begin{array}{l}\text { Follicular } \\
\text { pattern (-) }\end{array}$ & $P$ \\
\hline Isoechoic & 28 & 21 & & 20 & 29 & \\
\hline Hypoechoic & 4 & 2 & 0.65 & 2 & 4 & 0.72 \\
\hline Solid-cystic (mixed) & 3 & 1 & & 1 & 3 & \\
\hline Solid & 29 & 22 & 0.47 & 21 & 30 & 0.52 \\
\hline Halo (+) & 12 & 9 & & 9 & 12 & \\
\hline Halo (-) & 20 & 14 & 0.9 & 13 & 21 & 0.73 \\
\hline Microcalcification (+) & 6 & 7 & & 7 & 6 & \\
\hline Microcalcification (-) & 26 & 16 & 0.13 & 15 & 27 & 0.24 \\
\hline Peripheral blood supply & 24 & 16 & & 13 & 27 & \\
\hline Central blood supply & 8 & 7 & 0.65 & 9 & 6 & 0.064 \\
\hline Total & 32 & 23 & & 23 & 32 & \\
\hline
\end{tabular}

$P<0.05$ 


\section{DISCUSSION}

Thyroid nodules are more common in female patients $(F / M=4.5 / 1)(7)$. The sample in this study consisted of 55 patients, of whom 45 (81.8\%) were women. In a study conducted with 309 people, Kawamoto et al. noted that $90 \%$ of their cases were female (8), which was consistent with many other studies reporting that the females constituted the majority $(7,9)$.

In this study, a follicular pattern was detected in $3(9.67 \%)$ of 31 patients who underwent FNAB and 20 $(80.3 \%)$ of 24 patients who underwent surgery. Similar results were found compared with the literature. In a study, the authors discussed the results of other studies and stated that the rate of the follicular pattern in patients that who underwent surgery with suspected nodules on US was as follows: $38 \%$ among 96 patients in the study by Graves et al., $60 \%$ among 339 patients in the study by Deveci et al., $73 \%$ among 65 patients in the study by Wu et al., $60 \%$ among 25 patients in the study by Faquin et al., and $60 \%$ among 326 patients in the study by Yang et al. (10). In a study by Ugurluoglu et al., of the patients who underwent FNAB due to a total number of 1.096 follicular suspected nodules on US, SFN was cytopathologically detected in 5.8\% (11). The results of the present study were similar to the findings of the aforementioned studies.

It has been emphasized in various studies that no relationship exists between thyroid malignancy and nodule size. It has additionally been stated that the frequency of malignancy is considerably higher in nodules with a diameter of $<10 \mathrm{~mm}$. It has also been emphasized that no statistically significant difference is found between $<10 \mathrm{~mm}$ and $>10 \mathrm{~mm}$ nodules in terms of the prevalence of malignancy, and a cut-off value recommended for FNAB in nodules will not be beneficial $(12,13)$. In the present study, no statistically significant relationship was found between nodule size and pathology groups. Similarly, in a study conducted by Dogra et al. on 211 nodules, no difference in size was observed between the benign and malignant nodules. However, in a recent study on follicular neoplasia, it was suggested that nodule size and nodule volume might be the risk factors for follicular carcinoma (14). In the present study, the mean size of the nodules with a follicular pattern was calculated as $29.57 \mathrm{~mm}$, and the mean size of the nodules with a non-follicular pattern was calculated as $26.95 \mathrm{~mm}$. No statistically significant difference was found in the mean size of nodules between the patients with follicular and non-follicular patterns.

More malignancies are found in solid or largely solid nodules than in cystic nodules. In the literature, it has been emphasized that solid composition has a sensitivity rate of $69 \%-75 \%$ in detecting malignant nodules, but has a low positive predictive value ranging from 15.6 to $27 \%$ (15). Frates et al. and Dogra et al. found a relationship between solid content and malignancy $(16,17)$. However, the results of some studies are contradictory. In the present study, no statistically significant relationship was found between the pathology group and the nature of the nodule. In accordance with the literature, we concluded that nodule nature alone was not a sufficient criterion for the exclusion of malignancy $(7,16,18,20)$.

Some studies reported that the presence of an echo pattern was an important criterion for the differentiation of malignant and benign thyroid nodules and that the hypoechoic echo pattern was significant for the diagnosis of malignant nodules $(17,19,20)$. Dogra et al. reported that the hypoechoic echo pattern had a specificity of $41.7 \%$ and a sensitivity of $95.3 \%$ in detecting malignant nodules (21). Rahmani et al. detected a hypoechoic pattern in 27 of 38 malignant nodules and found a statistically significant relationship between hypoechoic pattern and malignant nodules. In the same study, the specificity of the hypoechoic echo pattern in differentiating malignant and benign nodules was determined as $69.4 \%$, sensitivity as $71 \%$, and negative predictive value as $78.8 \%$ (22). In the present study, no difference was found between the malignant and benign nodules in terms of nodule echogenicity. This might be related to the detection of an isoechoic pattern in most of the nodules evaluated in the sample. Appetecchia et al. emphasized that both hypoechoic and hyperechoic echo patterns were the most common sonographic appearances in malignant and benign nodules and they had similar rates of detection among malignant and benign nodules (7). In contrast, in a study conducted by Jeh et al., a hypoechoic pattern was found to be less $(35 \%)$ in follicular cancer, which was attributed to the differing amounts of colloids within the nodule (23). 
Many studies were conducted on the peripheral halo finding observed around the nodule. Most of them emphasized that the presence of a regular peripheral halo was generally observed in benign nodules, while an irregular incomplete peripheral halo is was observed more frequently in malignant lesions $(7,18,20)$. In the present study, no relationship was found between the absence of a peripheral halo and malignancy. In a study carried out by Rahmani et al., no peripheral halo was detected in 30 of 38 malignant nodules. In the same study, the sensitivity of the absence of a peripheral halo in detecting malignant nodules was shown to be $78.9 \%$ (22). However, Jeh et al., evaluating 150 patients diagnosed with papillary and follicular carcinoma, stated that a peripheral halo was also observed in malignant nodules. The authors attributed this result to follicular carcinomas presenting with a higher rate of complete peripheral halo, growth parallel to the thyroid parenchyma, and compression of normal tissue rather than invading normal tissue (23).

To date, many studies emphasized that the presence of microcalcification was a statistically significant criterion in detecting malignancy and had very high sensitivity and positive predictive values in diagnosing malignancy $(7,12,17)$. In the present study, no statistically significant relationship was found between the presence of microcalcification and malignant and benign nodules. Since microcalcification is small, posterior acoustic shading may not always be sufficiently visualized (17).

It has been suggested that the type of blood supply to the nodule also differs due to the different angiogenesis of benign and neoplastic nodules (24). Peripheral blood supply is mostly observed in benign lesions while a central, chaotic intranodular blood supply pattern is more common in malignant lesions $(9,24,25)$. Berni et al. stated that the Doppler US examination was not only non-invasive and cost-effective but also a reliable method for evaluating malignancy in cold nodules (25). An increase in size of a tumor depends on blood flow and angiogenesis. Angiogenesis and increased blood supply are expected findings in the malignant nodules of the thyroid. In the present study, no significant relationship was found between the type of blood supply to the nodule and malignancy, which might be due to the limited number of patients.

Comparing the pathology results obtained after FNAB and thyroidectomy on ultrasonographic examination of suspected nodules, the total malignancy rate was found to be $41.8 \%$, which was consistent with the literature. In a study conducted by Theoharis et al. with 89 cases, $48 \%$ of malignant pathologies were identified based on the results of FNAB applied to nodules suspected to be malignant (26). In other similar studies, the rate of malignant FNAB results was reported as $28 \%$ among 127 cases by Layfield et al., 13\% among 91 cases by Rabaglia et al., 19\% among 273 cases by Faquin and Baloch, and 20\% among 82 cases by Broome et al. (27-30). In the present study, among the 55 patients suspected to have follicular neoplasia based on thyroid US findings, a follicular pattern was pathologically observed in $40 \%$ and a malignant follicular pattern in $32 \%$, which was confirmed by cytopathological and histopathological diagnoses. This high rate of malignant pathological diagnosis in the present study could be explained by the US examination being undertaken by an experienced specialist in the center and operation decision based on multidisciplinary board evaluation of patients.

\section{CONCLUSIONS}

Thyroid ultrasonography, one of the noninvasive examination methods, plays an important role in the follow-up of thyroid cancer cases. Globally, to tackle this disease, the main objective is to define the nodules well, perform an ultrasonographic examination by an experienced operator, and follow up patients using the least invasive methods. The major limitation of the present study was its retrospective nature and a relatively small number of patients. However, it was considered that the findings of the present study and the published results in terms of the pathological diagnoses of malignancy and a follicular pattern were similar because the study was conducted in a highvolume center. Further studies with a larger number of patients are needed to determine the role of ultrasonographic evaluation in detecting the malignancy of suspicious nodules. 


\section{REFERENCES}

1. MahiraYunus, Zeba Ahmed. Significance of ultrasound features in predicting malignant solid throid nodules: Need for fineneedle aspiration. J Pak med Assoc 2010;60:848.

2. Lawrance Jr W, Kaplan BJ. Diagnosis and management of patients with thyroid nodules. J SurgOncol 2002;80:157-70.

3. Bagazzi F, Chiovata L. Role of conventional ultrasonography and colour flow-doppler sonography in predicting malignancy in 'cold' thyroid nodules. Eur J Endocrinol 1998;138: 41-46.

4. Park YJ, Kim JA. Thyroid nodules with macrocalcification: sonographic findings predictive of malignancy. Yonsei Med J 2014;55(2):339-344.

5. Haugen BR, Alexander EK. 2015 American Thyroid Association Management Guidelines for Adult Patients with Thyroid Nodules and Differentiated Thyroid Cancer. Thyroid 2016;26:1133.

6. Yong SS, Kim JH, Na DG. Ultrasonographic differentiation between nodular hyperplasia and neoplastic follicularpatterned lesions of the thyroid gland. Ultrasound in Med.\&Biol. 2016;42:1816-1824.

7. Appetecchia M, Solivetti FM. The association of colourdoppler sonography and conventional ultrasonography improves the diagnosis of thyroid carcinoma. Horm Res 2006;66:249-256.

8. Kawamoto $\mathrm{H}$, Koike E.Ultrasonographic characteristics of thyroid nodules: prediction of malignancy. Arch Surg. 2001;136:334-337.

9. Crescenzi A, Bianchini A. Risk of malignancy in non palpable thyroid nodules: predictive value of ultrasound and colourDoppler features. J ClinEndocrinolMetab 2002;87:1941-46.

10. Ugurluoglu C, Dobur F. Fine needle aspiration biopsy of thyroid nodules: cytologic and histopathologic correlation of 1096 patients. Int J ClinExpPathol 2015;8:11.

11. Gharib H, Papini E. American Association of clinical endocrinologists and associazionemediciendocrinologi medical guidelines for clinical practice for the diagnosis and management of the thyroid nodules. EndocrPract 2006;12:63102.

12. Chiferi V, De Nicola H. Flow pattern and vascular resistive index as predictors of malignancy risk in thyroid follicular neoplasms. J Ultrasound Med 2005;24:897-904.

13. Charboneau JW, Hay ID. Thyroid follicular carcinoma: sonographic features of 50 cases. AJR Am J Roentgenol 2010;194:44-54.

14. Charboneau JW, Hay ID. Sonography of thyroid nodules: a "classic pattern" diagnostic approach. Ultrasound Q 2005;21:157-165.

15. Kovaevic O, Skurla MS. Sonographic diagnosis of thyroid nodules: corelation with the results of sonographically guided fine-needle aspiration biopsy. J Clin. Ultrasound 2007;35:63-67.
16. Alexander E, Benson C. Prevalance and distribution of carcinoma in patients with solitary and multiple thyroid nodules on sonography. J ClinEndocrinolMetab. 2006;91:341117.

17. Hongdomnern G, Phuttharak W. Diagnosis performance of gray-scale versus combined gray-scale with colourdoppler ultrasonography in the diagnosis of malignancy in thyroid nodules. Asian Pac J Cancer Prev 2009;10:759-764.

18. Baek JH, Byun JS. Benign and malignant thyroid nodules: US differentiation.Multicenter retrospective study 1. Radiology 2008; 247:762-770.

19. Deveci MS, Deveci G. Fine-needle aspiration of follicular lesions of the thyroid.Diagnosis and follow-up. Cytojournal 2006;3:9.

20. Ahuja Al, Metreveli C. Ultrasound of thyroid nodules. Ultrasound Q 2000;16:111-122.

21. Behjati M, Firouznia K. Sonography and colourdoppler in the evaluation of cold thyroid nodules. Iran J Radiol 2004;14:13-16.

22. JehSK, Jung SL. Evaluating the degree of conformity of papillary carcinoma and follicular carcinoma to the reported ultrasonographic findings of malignant thyroid tumor. Korean J Radiology 2007;27:847-860.

23. Farrell S, Hoang JK. US features of thyroid malignancy: pearls and pitfalls. Radiographics 2007; 27:847-860.

24. Brkljacic B, Ivanac K. Vascularization of benign and malignant thyroid nodules: CD US evaluation. Ultraschall Med 2007;28:502-506.

25. Gritzmann N, Koischwitz D. Sonography of the thyroid and parathyroid glands. RadiolClin North Am 2000;38:1131-1144.

26. Theoharis CG, Schofield KM. The Bethesda thyroid fineneedle aspiration classification system: year 1 at an academic institution. Thyroid 2009;19:1215-1223.

27. Layfield LJ, Morton MJ. Implications of the proposed thyroid fine-needle aspiration category of 'follicular lesion of undetermined significance':a five year multi-institutional analysis. DiagnCytopathol 2009;37:710-714.

28. Rabaglia JL, Kabbani W. Effect of the Bethesda system for reporting thyroid cytopathology on thyroidectomy rates and malignancy risk in cytologically indeterminate lesions. Surgery 2010;148:1267-1272.

29. Faquin WC, Baloch ZW. Fine-needle aspiration of follicular patterned lesions of the thyroid: Diagnosis, management, and follow up according to National Cancer Institute $(\mathrm{NCl})$ recommendations. DiagnCytopathol 2010;38:731-739.

30. Broome JT, Solorzano CC. The impact of atypia/follicular lesion of undetermined significance on the rate of malignancy in thyroid fine-needle aspiration: evaluation of the Bethesda System for Reporting Thyroid Cytopathology. Surgery 2010;150:1234-1241. 